\title{
Hepatitis B viral core protein disrupts human host gene expression by binding to promoter regions
}

\author{
Yanhai Guo ${ }^{1 *+}$, Wei Kang ${ }^{2 \dagger}$, Xiaoying Lei ${ }^{1+}$, Yongnian $\mathrm{Li}^{3}$, An Xiang ${ }^{1}$, Yonglan Liu' ${ }^{1}$ Jinrong Zhao ${ }^{1}$, Ju Zhang ${ }^{1}$
} and Zhen Yan ${ }^{1}$

\begin{abstract}
Background: The core protein $(\mathrm{HBC})$ of hepatitis B virus ( $\mathrm{HBV}$ ) has been implicated in the malignant transformation of chronically-infected hepatocytes and displays pleiotropic functions, including RNA- and DNA-binding activities. However, the mechanism by which $\mathrm{HBC}$ interacts with the human genome to exert effects on hepatocyte function remains unknown. This study investigated the distribution of $\mathrm{HBC}$ binding to promoters in the human genome and evaluated its effects on the related genes' expression.
\end{abstract}

Results: Whole-genome chromatin immunoprecipitation microarray (ChIP-on-chip) analysis was used to identify $\mathrm{HBC}$-bound human gene promoters. Gene Ontology and pathway analyses were performed on related genes. The quantitative polymerase chain reaction assay was used to verify ChIP-on-chip results. Five novel genes were selected for luciferase reporter assay evaluation to assess the influence of $\mathrm{HBC}$ promoter binding. The HBc antibody immunoprecipitated approximately 3100 human gene promoters. Among these, 1993 are associated with known biological processes, and 2208 regulate genes with defined molecular functions. In total, 1286 of the related genes mediate primary metabolic processes, and 1398 encode proteins with binding activity. Sixty-four of the promoters regulate genes related to the mitogen-activated protein kinase (MAPK) pathways, and 41 regulate Wnt/beta-catenin pathway genes. The reporter gene assay indicated that $\mathrm{HBC}$ binding up-regulates proto-oncogene tyrosine-protein kinase (SRC), type 1 insulin-like growth factor receptor (IGF1R), and neurotrophic tyrosine kinase receptor 2 (NTRK2), and down-regulates v-Ha-ras Harvey rat sarcoma viral oncogene (HRAS).

Conclusion: $\mathrm{HBC}$ has the ability to bind a large number of human gene promoters, and can disrupt normal host gene expression. Manipulation of the transcriptional profile in HBV-infected hepatocytes may represent a key pathogenic mechanism of HBV infection.

Keywords: Hepatitis B virus, Hepatitis B core protein, Chromatin immunoprecipitation microarray, ChIP-on-chip, Gene expression, DNA-protein interaction

\section{Background}

Hepatitis B virus (HBV) infection is a serious public health threat. Worldwide, more than 400 million people are infected with HBV. Chronic infection is associated with a high risk of potentially life-threatening liver diseases, including hepatitis, cirrhosis, and hepatocellular carcinoma [1,2]. However, despite extensive research efforts, the molecular mechanisms of chronic HBV

\footnotetext{
* Correspondence: gyanhai@fmmu.edu.cn

${ }^{\dagger}$ Equal contributors

'State Key Laboratory of Cancer Biology, Department of Pharmacogenomics, School of Pharmacy, Fourth Military Medical University, 169 West Changle Road, Xi'an 710032, China

Full list of author information is available at the end of the article
}

infection remain to be fully elucidated. HBV, a hepatotropic virus, is a $3.2 \mathrm{~kb}$ partially double-stranded DNA virus that replicates through the reverse transcription of pre-genomic RNA. Its DNA genome contains four overlapping open reading frames (ORFs), which encode the surface antigen-associated $\mathrm{S}$ proteins (large $\mathrm{S}$, middle $\mathrm{S}$, and major $S$ ), core antigen proteins (precore and core $(\mathrm{HBc}))$, reverse transcriptase $(\mathrm{P})$, and the multifunctional $\mathrm{X}$ protein $(\mathrm{HBx})$ [3]. Diverse molecules and proteins may participate in HBV-infection induced chronic hepatitis. For example, a recent study indicated that the cytokines lymphotoxin (LT) alpha and beta and their receptor (LTbetaR) are upregulated in $\mathrm{HBV}$-induced hepatitis and hepatocellular carcinoma (HCC) [4]. While

\section{Biomed Central}


the HBV proteins play essential roles in viral processes, such as virulence and replication, they also exert direct effects on host cellular functions to promote virus survival. For instance, HBV targets host genes that are involved in cell survival, and while this facilitates escape from immune surveillance and clearance, it also favors malignant transformation [5].

One of the possible mechanisms that contributes to malignant transformation involves HBV-encoded transactivating factors, which influence particular intracellular signal transduction pathways by altering the host gene expression profile in hepatocytes [6]. Several HBV protein products have been characterized as modulators of cellular growth, repair, and death, all of which are involved in oncogenesis [7]. Studies have shown that $\mathrm{HBx}$ can transactivate the expression of all HBV proteins, including $\mathrm{HBc}$, which acts as the core antigen that stimulates the human immune response. The $21-22 \mathrm{kDa}$ $\mathrm{HBc}$ protein has been detected in both the nuclear and cytoplasmic compartments of hepatocytes infected by HBV $[8,9]$. Subsequent studies have revealed pleiotropic functions of $\mathrm{HBc}$ that affect host processes, including the malignant transformation of chronically infected liver cells [10]. Moreover, this newly-recognized dual character of $\mathrm{HBc}$ as a novel regulator of the HBV life cycle and of hepatocellular carcinogenesis has been hypothesized to involve $\mathrm{HBx}$, possibly through an inhibitory feedback mechanism [11]. HBc was shown to repress the expression of the human tumorigenesisassociated genes, interferon (IFN)- $\beta$ and p53 [12]; the latter of which is also modulated by $\mathrm{HBx}$, via binding to the encoded protein and suppressing its activity [13]. Additionally, recent studies have suggested that HBV down-regulates the human IFN-inducible MxA promoter through direct interaction of precore/core proteins [14], and have shown that $\mathrm{HBc}$ inhibits apoptosis induced by the tumor necrosis factor family member, TRAIL, in hepatocytes by blocking gene expression of the pathway-related death receptor [10]. Collectively, these studies indicate that $\mathrm{HBc}$ may interact with the human genome to modulate normal functions of liver cells infected HBV.

$\mathrm{HBc}$ is the major capsid protein of the virus and selfassembles to form the subviral 30-32 nm nucleocapsid particles, which package the viral polymerase and pregenomic RNA during RNA replication. The HBc carboxy-terminus contains two nuclear localization signals in regions of arginine-rich sequences, and an SPRRR motif with several serine residues that are targets of phosphorylation [15]. In general, the C-terminus amino acid sequence is rich in basic amino acid residues, such as arginine and lysine, and resembles the structure of a mammalian DNA binding protein, protamine [16,17]. Although $\mathrm{HBc}$ has been sufficiently demonstrated by many studies to functionally bind to both virus- and hostderived RNA and DNA, the way in which $\mathrm{HBc}$ interacts with the human genome to modulate normal hepatocyte function in HBV infection remains unknown. Based on the findings of in vivo studies that showed core particles binding specifically to the HBV pregenome and genome, we hypothesized that $\mathrm{HBc}$ may also bind specifically to certain human gene promoters, either through its Cterminal functional domain or its $\mathrm{N}$-terminal assembling domain.

A recently developed high-throughput strategy to perform targeted or genome-wide studies of transcription binding factors is the chromatin immunoprecipitation (ChIP)-coupled DNA microarray analysis. Known as ChIP-on-chip, this technique couples immunoprecipitation of chromatin-bound transcription factors with the identification of bound DNA sequences through hybridization on DNA microarrays [18]. In the present work, we used a combination of ChIP and location analysis with genome-wide tiling arrays to generate a human genome-wide binding profile of $\mathrm{HBc}$. The human genes whose promoters were bound by $\mathrm{HBc}$ and had functions related to tumorigenesis were selected for verification by quantitative PCR (qPCR) and functional analysis by gene expression assays. Identification of the human gene targets of the $\mathrm{HBV}$-encoded $\mathrm{HBc}$ protein provides further insights into $\mathrm{HBV}$ pathogenesis and potential new targets of molecular therapeutics against HBV-associated hepatocellular carcinogenesis.

\section{Methods}

\section{Research subjects and biological samples}

Thirteen unrelated patients, including nine males and four females ranging in age from 19 to 55 years-old (mean: 33.5), diagnosed with chronic hepatitis B (CHB) were recruited to our study. Three male healthy blood donors, whose age and gender matched the patient group, were enrolled as controls. The diagnostic criteria of CHB was based on the combination of clinical history, physical examination, imaging and laboratory data, and/ or histology, according to the published guidelines of the Chinese Medical Association [19]. All patients were also evaluated to ensure that the following criteria were satisfied: (1) serum hepatitis B surface antigen (HBsAg), hepatitis $\mathrm{B}$ e antigen ( $\mathrm{HBeAg})$, and hepatitis $\mathrm{B}$ core antibody ( $\mathrm{HBcAb})$ (IgG) positive, but hepatitis B surface antibody (HBsAb) and hepatitis $\mathrm{B}$ e antibody (HBeAb) negative; (2) $\mathrm{HBsAg}$ and $\mathrm{HBcAb}$ positivity for over 12 months; (3) HBV DNA $\geq 3 \log 10$ copies/mL; (4) at least a six-month history of no type of antiviral, immuno-suppressive, or immunomodulatory treatment; (5) no evidence of co-infection with human immunodeficiency virus, hepatitis $\mathrm{A}$ virus, hepatitis $\mathrm{C}$ virus, or 
hepatitis D virus; (6) no routine alcohol consumption, by self-report.

A percutaneous liver biopsy was obtained from all study participants by using a $16 \mathrm{G}$ Tru cut soft tissue biopsy needle. A $\sim 176 \mathrm{mg}$ specimen of normal liver tissue was obtained from the three healthy controls. A 9.3 $19.0 \mathrm{mg}$ total weight specimen of benign liver tissue was obtained from each CHB patient through a sequence of two to four biopsies. All liver samples were evaluated by histological examination, and stored in liquid nitrogen until further use. The clinical characteristics of patients and their corresponding biological samples are listed in Table 1. All subjects provided written informed consent to participate in the study, and the experimental protocol was approved by the Ethics Committees of the 323 Hospital and Affiliated Hospital of Xi'an Medical College (both of Xi'an, China).

\section{Chromatin immunoprecipitation microarrays and data analysis}

The hepatocytes isolated from pooled tissue samples ( $\mathrm{CHB}, \mathrm{n}=4$; control, $\mathrm{n}=3$ ) were cross-linked with formaldehyde and subjected to the standard ChIP procedure, as described elsewhere [20]. Briefly, the cross-linked hepatocytes were lysed, and nuclear extracts were sonicated to solubilize the protein-DNA complexes. The supernatants were then incubated overnight with mouse antibodies against the HBV core protein or normal mouse IgG (negative control) (both from Santa Cruz
Biotechnology, Santa Cruz, CA, USA). The immunocomplexes were precipitated by addition of protein G-Sepharose (GE Healthcare, formerly Amersham Biosciences, Picastaway, NJ, USA), and eluted by incubation with the corresponding elution buffer. The cross-links were reversed by heating at $65^{\circ} \mathrm{C}$ for $5 \mathrm{~h}$. The released proteins were digested with proteinase $\mathrm{K}$, and the precipitated chromatin was extracted using a standard phenol-chloroform method.

The precipitated chromatin samples were sent to Kangcheng Biological Engineering Ltd., Comp. (Shanghai, China) for hybridization to the NimbleGen HG18 RefSeq Promoter Microarray (Roche Nimblegen, Madison, WI, USA). The immunoaffinity-enriched DNA was amplified for labeling using the GenomePlex ${ }^{\circledR}$ Whole Genome Amplification kit (Sigma-Aldrich, St. Louis, MO, USA), according to the manufacturer's protocol. The amplified DNA samples were purified with a QIAquick spin kit (Qiagen, Valencia, CA, USA). The purified DNA was quantified using a Nanodrop ND-1000 spectrophotometer (Nanodrop Technologies, Wilmington, DE, USA). For DNA labeling, as previously described [21], the NimbleGen Dual-Color DNA labeling kit was used, according to the manufacturer's guidelines detailed in the NimbleGen ChIP-on-chip protocol. A permutation-based peak-finding algorithm, NimbleScan (v2.5; NimbleGen), was used to identify peaks that represent significant positive enrichment. NimbleScan detects peaks by searching for four or more probes producing signals that were above the

Table 1 Clinical characteristics of study participants and their corresponding biological samples

\begin{tabular}{|c|c|c|c|c|c|c|c|}
\hline \multicolumn{2}{|c|}{ Number } & \multirow[t]{2}{*}{ Sex } & \multirow[t]{2}{*}{ Age } & \multirow[t]{2}{*}{ Disease $^{* *}$} & \multirow[t]{2}{*}{ HBV DNA (copies/mL) } & \multicolumn{2}{|c|}{ Tissue weight (mg) } \\
\hline Pool* & Samples & & & & & Sample & Pool \\
\hline \multirow[t]{3}{*}{1} & 1 & male & 30 & $\mathrm{CHB}$ & $4.5 \times 10^{5}$ & 16.8 & 42.1 \\
\hline & 2 & male & 27 & $\mathrm{CHB}$ & $5.7 \times 10^{6}$ & 16.0 & \\
\hline & 3 & male & 31 & $\mathrm{CHB}$ & $4.2 \times 10^{6}$ & 9.3 & \\
\hline \multirow[t]{3}{*}{2} & 4 & male & 21 & $\mathrm{CHB}$ & $3 \times 10^{5}$ & 17.0 & 43.6 \\
\hline & 5 & male & 34 & $\mathrm{CHB}$ & $7.5 \times 10^{5}$ & 12.1 & \\
\hline & 6 & male & 25 & $\mathrm{CHB}$ & $3.2 \times 10^{6}$ & 14.5 & \\
\hline \multirow[t]{3}{*}{3} & 7 & male & 40 & $\mathrm{CHB}$ & $3 \times 10^{4}$ & 14.3 & 42.1 \\
\hline & 8 & male & 39 & $\mathrm{CHB}$ & $8.3 \times 10^{5}$ & 14.8 & \\
\hline & 9 & male & 35 & $\mathrm{CHB}$ & $1.34 \times 10^{6}$ & 13.0 & \\
\hline \multirow[t]{4}{*}{4} & 10 & female & 43 & $\mathrm{CHB}$ & $3.6 \times 10^{5}$ & 10.9 & 48.6 \\
\hline & 11 & female & 45 & $\mathrm{CHB}$ & $3.7 \times 10^{7}$ & 17.8 & \\
\hline & 12 & female & 40 & $\mathrm{CHB}$ & $6.9 \times 10^{7}$ & 9.7 & \\
\hline & 13 & female & 41 & $\mathrm{CHB}$ & $4.2 \times 10^{6}$ & 10.2 & \\
\hline \multirow[t]{3}{*}{5} & 14 & male & 31 & control & undetected & 81.0 & 176.0 \\
\hline & 15 & male & 30 & control & undetected & 50.0 & \\
\hline & 16 & male & 25 & control & undetected & 45.0 & \\
\hline
\end{tabular}

* Liver biopsy samples from three to four individuals were pooled into a single specimen for ChIP-on-chip and ChIP-qPCR analyses.

** $\mathrm{CHB}$, chronic hepatitis B. The serum HBV markers used for diagnosis were: $\mathrm{HBsAg}(+), \mathrm{HBsAb}(-), \mathrm{HBeAg}(+), \mathrm{HBeAb}(-)$, and anti-HBc(+). Healthy controls were confirmed as: $\operatorname{HBsAg}(-), \operatorname{HBsAb}(-), \operatorname{HBeAg}(-), \operatorname{HBeAb}(-)$, and anti-HBc(-). 
specified cutoff values (ranging from $90 \%$ to $15 \%$ ) in a given $500 \mathrm{bp}$ sliding window. The cutoff values were defined as a percentage of a hypothetical maximum, which was the mean +6 [standard deviation]. The ratio data was then randomized 20 times to evaluate the probability of "false positives". Each peak was then assigned a false discovery rate (FDR) score based on the randomization. The lower the FDR score, the more likely the peak was considered to correspond to a protein binding site.

To monitor the possible interference by non-specific hybridization signals of HBV DNA and promoter microarray probes, the amplified PCR products of HBV DNA genomic sequences were hybridized to the promoter microarray probes. HBV DNA was extracted from the serum (HBV DNA $6.0 \times 10^{8}$ copies $/ \mathrm{mL}$ ) of HBV-infected patients using the standard phenol-chloroform method. Six overlapping regions of HBV genomic sequence were amplified by PCR, and the length of these products ranged from $534 \mathrm{bp}$ to $654 \mathrm{bp}$. The sequences represent the entire HBV genome, with each two neighboring fragments overlapping by 79-99 bp of sequence (see Additional file 1). The PCR products were sent to Kangcheng Co. for hybridization to the NimbleGen HG18 RefSeq Promoter Microarray.

\section{Quantitative polymerase chain reaction (qPCR)}

The results from the ChIP-on-chip microarray analysis were verified by qPCR. The reaction was carried out using an iCycler iQ real-time PCR detection system and the $\mathrm{iQ}$ SYBR Green Supermix detection reagent (BioRad, Hercules, CA, USA). Gene-specific primers were designed with the Beacon Designer software (Premier Biosoft International, Bio-Rad) and are listed in Additional file 2. Relative expression was normalized as a percentage of input (total chromosomal DNA). To account for chromatin sample preparation differences, each output DNA fraction Ct value was normalized to the input DNA fraction $\mathrm{Ct}$ value for the same qPCR assay $(\Delta \mathrm{Ct})$. Relative quantification was performed by the Livak $\left(2^{-\Delta \Delta C t}\right)$ method [22]. The \% input for each output fraction was calculated according to the formula: $\%$ Input $=[2($ CtInput-CtOutput $) \times \mathrm{Fd}] \times 100 \%$, where $\mathrm{Fd}$ is the input dilution factor).

\section{Luciferase reporter assay}

Genomic fragments (250 $500 \mathrm{bp}$ ) encompassing a single $\mathrm{HBc}$ peak from the promoter region of selected genes were amplified by PCR from human genomic DNA and cloned into pGL3-basic expression vectors (Promega, Madison, WI, USA) (see Additional file 3). The HBc expression plasmid (pUSC) was constructed as previously described [23], for use in co-transfection assays. The inserted sequences of all reporter constructs were verified by direct sequencing.
HepG2 cells were transfected with $1000 \mathrm{ng}$ of one of the pGL3-basic constructs plus $800 \mathrm{ng}$ of the pUSC plasmid, together with $20 \mathrm{ng}$ pRL-TK vector (Promega) as an internal control for transfection efficiency, using the JetPEI transfection reagent (PolyPlus-Transfection Co., Illkirch, France). After 24 hours, cells were collected and luciferase activities were measured using the Dual-Luciferase Reporter Assay system (Promega). Firefly luciferase activities were normalized to the level of Renilla luciferase activity in each transfection experiment. Relative luciferase levels were expressed as the average of three independent experiments performed in quadruplicate.

\section{Data analysis}

Raw microarray data were extracted as pair files by the NimbleScan software. We performed median-centering, quantile normalization, and linear smoothing by using the Bioconductor packages, Ringo, Limma, and MEDME. The gene ontology (GO) and pathway analysis of promoter-related genes were carried out online (http:// www.geneontology.org). Gene pathway analysis was conducted with the Kyoto Encyclopedia of Genes and Genomes (KEGG) collection of online databases dealing with genomes, enzymatic pathways, and biological chemicals [24].

Statistical analysis was performed using SPSS software (v9.0; SPSS Inc., Chicago, IL, USA). Statistically significant differences among continuous variables between two independent groups were analyzed by the MannWhitney test. A $p$-value less than 0.05 was considered statistically significant.

\section{Results}

\section{Genome-wide binding profile of $\mathrm{HBC}$ in $\mathrm{HBV}$-infected hepatocytes}

We performed ChIP-on-chip analysis with the NimbleGen HG18 RefSeq Promoter Microarray consisting of 18028 of the best defined human transcript-associated promoters. Hepatocytes infected with HBV and expressing HBV core protein were isolated from $\mathrm{CHB}$ patients, immunoprecipitated with $\mathrm{HBc}$ antibody, and investigated to determine the genome-wide profile of bound host gene promoters. IgG pre-incubated with 100-fold excess HBc antibody was used as a negative control, and immunoprecipitation of hepatocyte cells from healthy individuals was used as a blank control. Meanwhile, no non-specific hybridization signals between the HBV DNA and the chip probes (NimbleGen HG18 RefSeq Promoter Microarray) were detected. The HBV-infected hepatocyte-specific enrichment that was achieved by sequential immunoprecipitations was expected to facilitate a sufficiently comprehensive identification of $\mathrm{HBc}$ binding sites, including the relatively weakly bound sites. 
In total, $3100 \mathrm{HBc}$-immunoprecipitated host promoter regions were found to be enriched by at least 2 -fold in $\mathrm{HBc}$-infected tissues compared to the blank controls ( $p$ $<0.05$ ) (see Additional file 4). Among these, 2607 (84.10\%) were located in high CpG density promoters (HCPs), 356 (11.50\%) in intermediate CpG density promoters (ICPs), and 137 (4.42\%) in low CpG density promoters (LCPs). Ninety-two percent (2833) of the differentially enriched gene promoter regions were immunoprecipitated from all four of the pooled samples evaluated. These results suggest that the HBV core protein might bind to a broad spectrum of human gene promoters, especially those featuring high CpG density.

The GO analysis is a powerful tool by which the 3100 genes associated with the immunoprecipitated promoters may be correlated with biological processes or known protein functions. The GO annotations for biological processes were found for 1933 of the genes related to the immunoprecipitated promoters $(p=1.1 \mathrm{E}$ 08 ), and annotations for molecular functions were found for $2022(p=6.4 \mathrm{E}-11)$. The biological processes represented within this dataset of putative $\mathrm{HBc}$ targets included: metabolic process $(1404 ; p=3.0 \mathrm{E}-08)$; primary metabolic process $(1286 ; p=1.1 \mathrm{E}-08)$; cellular metabolic process $(1248 ; p=4.1 \mathrm{E}-08)$; and biological regulation (1206; $p=1.7 \mathrm{E}-03)$ (Figure 1). In addition, a remarkable amount of genes were found to be associated with dsRNA fragmentation, and intermediate filament cytoskeleton organization (Figure 2); the fold-enrichment value for these two particular groups of genes was 3.495 $(p=1.5 \mathrm{E}-03)$ and $3.0749(p=1.0 \mathrm{E}-03)$, respectively. The ten molecular functions of the encoded gene products that were most represented within the dataset of putative $\mathrm{HBc}$ targets are listed in Table 2. Protein binding was associated with the largest amount of putative
HBc targets (1398, $p=9.7 \mathrm{E}-13)$, followed by catalytic activity $(881, p=2.9 \mathrm{E}-06)$ and transcription regulator activity $(289, p=2.4 \mathrm{E}-06)$. Further analysis suggested that one of the highly enriched gene promoters was related to an encoded protein product with MAP kinase phosphatase activity and another one was related to a protein with acylglycerol O-acyltransferase activity; the foldenrichment value of these two was, respectively, 3.5303 $(p=1.4 \mathrm{E}-03)$ and $3.4967(p=6.8 \mathrm{E}-04)$ (Figure 3$)$.

Pathway analysis was performed for all genes related to the promoters that immunoprecipitated with the HBV core protein. The 3100 genes were found to represent 55 pathways of the KEGG, according to the molecular interactions, reactions, and relations of the genes (see Additional file 5). Table 3 lists the top ten pathways corresponding to the significantly enriched promoters. The Enrichment score for pathways involved in tumor pathogenesis, MAPK signaling and Wnt signaling, were $8.5732(p=2.7 \mathrm{E}-09)$ and $7.5542(p=2.8 \mathrm{E}-08)$, respectively (Figure 4).

In order to verify the reliability of the ChIP-on-chip results, qPCR was used to detect the promoter sequences of selected genes in ouput DNA fractions from $\mathrm{CHB}$ patients and healthy controls. Twelve genes were selected for this analysis based upon known functions in tumor pathogenesis, and included: fibroblast growth factor 4 (FGF4), v-Ha-ras Harvey rat sarcoma viral oncogene (HRAS), mitogen-activated protein kinase kinase 2/MEK2 (MAP2K2), neurotrophic tyrosine kinase receptor 2 (NTRK2), platelet-derived growth factor A chain (PDGFA), platelet-derived growth factor B chain (PDGFB), Ras guanine nucleotide-releasing factor (RASGRF2), wingless-type MMTV integration site family (WNT11, a member of the Wnt superfamily of secreted glycoproteins), type 1 insulin-like growth factor receptor

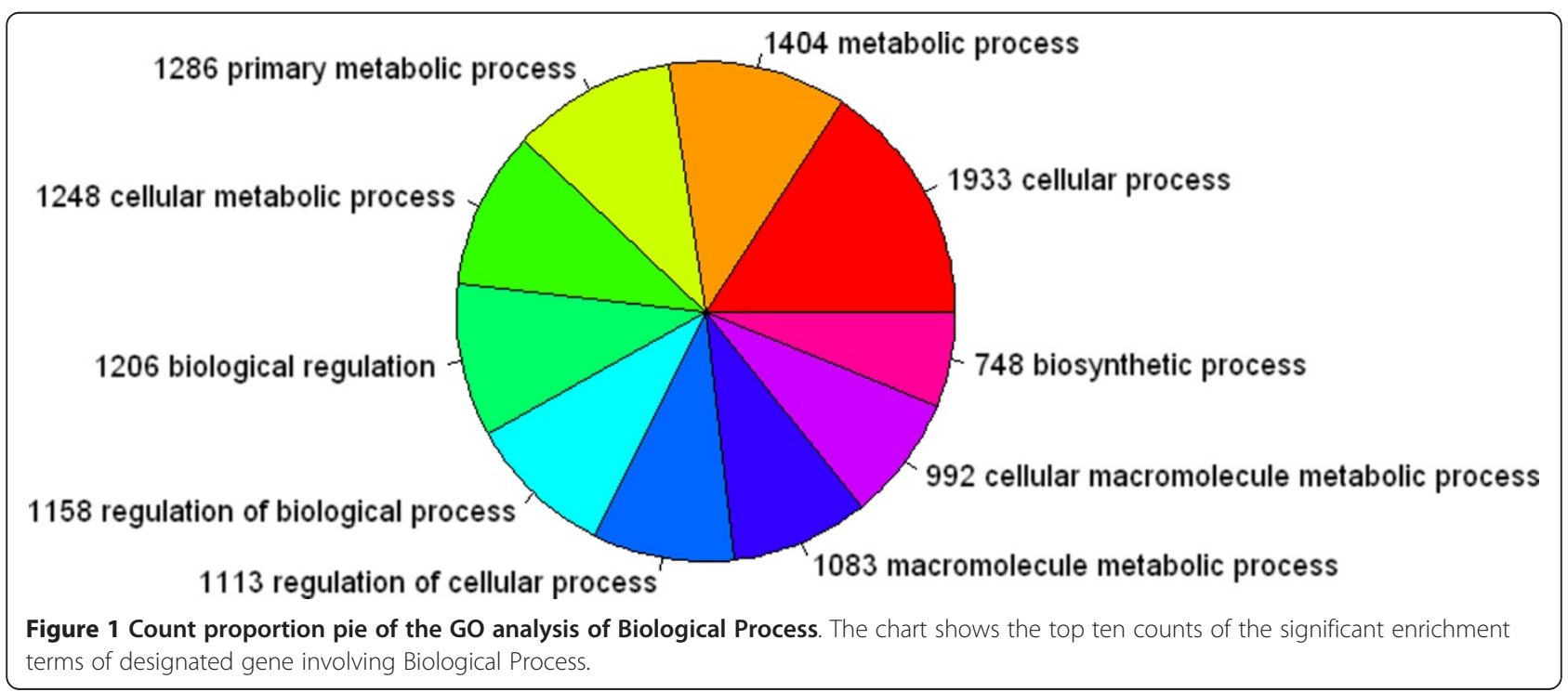




\section{cellular response to dsRNA}

production of small RNA involved in gene silencing by RNA

dsRNA fragmentation

axon cargo transport

production of miRNAs involved in gene silencing by miRNA

substrate-bound cell migration

glycosphingolipid biosynthetic process

intermediate filament-based process

intermediate filament cytoskeleton organization

gut morphogenesis

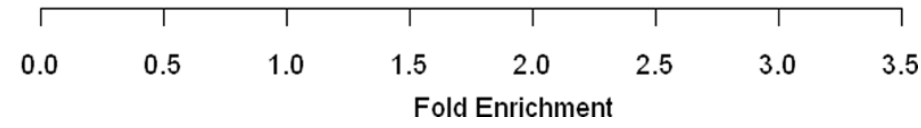

Figure 2 The bar plot of the GO analysis of Biological Process. The bar plot shows the top ten Fold Enrichment value of the significant enrichment terms of designated gene involving Biological Process.

(IGF1R), proto-oncogene tyrosine-protein kinase (SRC), vascular endothelial growth factor-B (VEGFB), and vascular endothelial growth factor-C (VEGFC). The results indicated that the promoters of these 12 genes $(p<0.05)$ were immunoprecipitated by anti-HBc, suggesting that $\mathrm{HBc}$ physically interacted with each. Moreover, we tested the promoter sequences that were negative in $\mathrm{HBc}$-ChIPchip screening, such as the promoter of the tumor protein 53 (p53) gene. As shown in Figure 5, the highest percent of output to input (\% Input) occurred with IGF1R

Table 2 The top ten molecular functions associated with HBc-immunoprecipitated host promoter sequences

\begin{tabular}{llll}
\hline Molecular function & Count* $^{*}$ & $\boldsymbol{p}_{\text {-value }}^{* *}$ & FDR $^{* * *}$ \\
\hline Binding & 2022 & $6.4000 \mathrm{E}-11$ & $2.5632 \mathrm{E}-08$ \\
Protein binding & 1398 & $9.7000 \mathrm{E}-13$ & $7.7697 \mathrm{E}-10$ \\
Catalytic activity & 881 & $2.8000 \mathrm{E}-06$ & $2.8035 \mathrm{E}-04$ \\
lon binding & 702 & $9.4800 \mathrm{E}-03$ & $1.3559 \mathrm{E}-01$ \\
Cation binding & 697 & $4.9800 \mathrm{E}-03$ & $8.3437 \mathrm{E}-02$ \\
Metal ion binding & 692 & $4.2500 \mathrm{E}-03$ & $8.3030 \mathrm{E}-02$ \\
Nucleotide binding & 392 & $4.9000 \mathrm{E}-04$ & $2.0657 \mathrm{E}-02$ \\
Purine nucleotide binding & 339 & $6.2000 \mathrm{E}-04$ & $02.4831 \mathrm{E}-02$ \\
Transferase activity & 337 & $2.0000 \mathrm{E}-08$ & $5.3400 \mathrm{E}-06$ \\
Ribonucleotide binding & 328 & $3.6000 \mathrm{E}-04$ & $1.7434 \mathrm{E}-02$ \\
Transcription regulator activity & 289 & $2.4000 \mathrm{E}-06$ & $2.7463 \mathrm{E}-04$ \\
\hline
\end{tabular}

* Number of genes associated with the corresponding molecular function

** Significance testing value of genes associated with the corresponding molecular function.

*** False discovery rate of genes associated with the corresponding molecular function, as determined by the Benjamini \& Hochberg (1995) testing method.
(34.3\%), followed by NTRK2 (27.9\%), HRAS (26.8\%), SRC (22.0\%), VEGFC (19.0\%), PDGFA (17.7\%), FGF4 (14.4\%), VEGFB (14.2\%), WNT11 (14.0\%), PDGFB (12.1\%), MAP2K2 (10.2\%), and RASGRF2 (9.4\%). In addition, qPCR analysis further confirmed that $\mathrm{HBc}$ did not bind to the p53 promoter, as the p53\% input was negative.

\section{HBV core protein binding to host gene promoters} disrupts normal cellular gene expression

The above results suggested that HBV core protein can bind to host gene promoters, especially those involved in tumor pathogenesis. To investigate whether $\mathrm{HBC}$ binding regulated the transcriptional activity of these host cellular genes, reporter-gene expression assays were carried out. The promoter sequences of five genes (HRAS, NTRK2, PDGFA, IGF1R, and SRC) with $>15 \%$ of output to input, and the promoter sequences of p53 as a negative control were selected for cloning into expression vectors and subsequent analysis. The sequences of the above promoters are listed in Additional file 3. As shown in Figure 6, the expression of all genes, with the exception of PDGFA and p53, was significantly changed in the presence of $\mathrm{HBc}$, as evidenced by differential luciferase activity in $\mathrm{HBc}$ co-transfected cells and cells without the $\mathrm{HBc}$ expression vector. For SRC, IGF1R, and NTRK2, HBc co-transfection led to significant greater gene expression (luciferase activity fold-change: SRC, 3.5; IGF1R, 2.7, NTRK2, 2.1; $p<0.05)$. In contrast, HBc co-transfection led to significantly less expression of HRAS (luciferase activity fold-change: $0.4 ; p<0.05$ ). The 
MAP kinase phosphatase activity

MAP kinase tyrosine/serine/threonine phosphatase activity

acylglycerol O-acyltransferase activity

protein phosphatase type $2 \mathrm{~A}$ regulator activity

insulin-like growth factor binding

UDP-galactosyltransferase activity

acetylgalactosaminyltransferase activity

O-acyltransferase activity

galactosyltransferase activity

microtubule binding

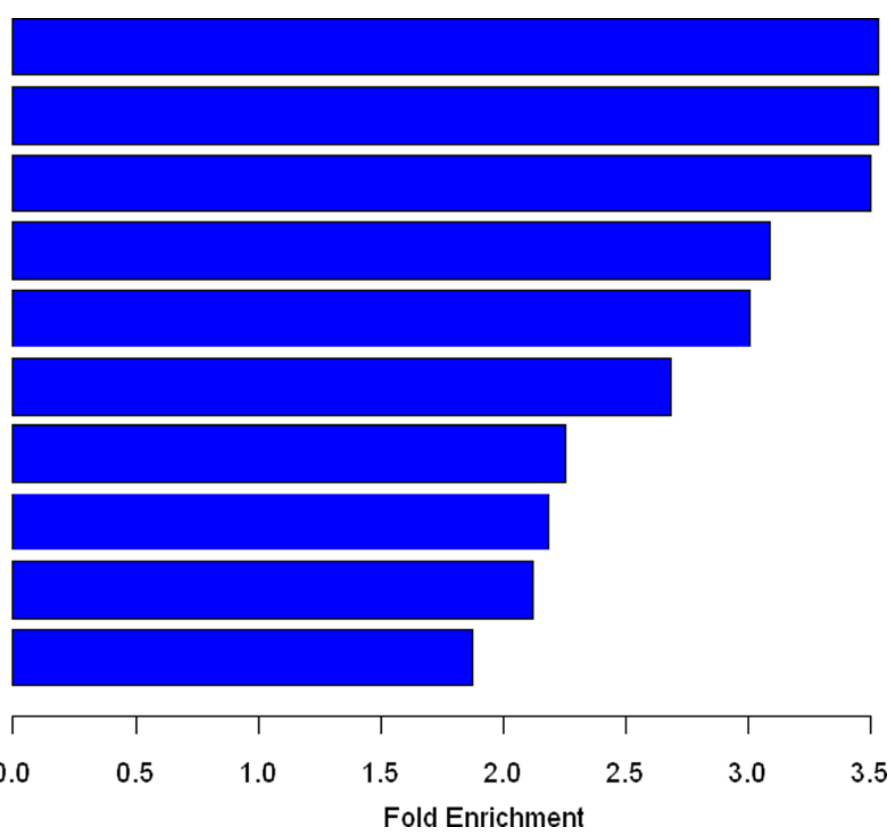

Figure 3 The bar plot of the GO analysis of Molecular Function. The bar plot shows the top ten Fold Enrichment value of the significant enrichment terms of designated gene involving Molecular Function.

HBc co-transfection slightly decreased the transcriptional activity of the PDGFA and p53 genes, whereas no statistical differences were detected (luciferase activity fold-change: PDGFA, 0.9, p > 0.05; P53, 0.9, $\mathrm{p}>0.05$ ).

\section{Discussion}

In our study, we used the GenomePlex ${ }^{\circledR}$ Whole Genome Amplification ChIP-chip analyses and found that HBC can bind to more than three-thousand promoters in the human genome. Among this set of gene promoters, the high $\mathrm{CpG}$ density promoters were the most common. By evaluating the corresponding the $\mathrm{GO}$ annotations and the KEGG pathways for genes affiliated with each of these promoters, we determined that $\mathrm{HBc}$ tended to target the regulatory regions of genes with molecular function and malignant transformation in the liver cell repertoire. These results were consistent with our previous data showing that $\mathrm{HBc}$ preferentially binds CpG islands within the HBV covalently closed circular DNA [25]. To our knowledge, our current study is the first to provide evidence that $\mathrm{HBc}$ can bind to a large number of host gene promoters throughout the whole human

Table 3 The top ten pathways associated with HBc-immunoprecipitated host promoter sequences with the highest enrichment scores

\begin{tabular}{lclll}
\hline Pathway & Selection counts* & $\boldsymbol{p}^{*}$ value $^{* *}$ & FDR $^{* * *}$ & Enrichment score $^{* * * *}$ \\
\hline MAPK signaling & 64 & $2.6720 \mathrm{E}-09$ & $5.8516 \mathrm{E}-07$ & 8.5732 \\
Wnt signaling & 41 & $2.7910 \mathrm{E}-08$ & $3.0561 \mathrm{E}-06$ & 7.5542 \\
Melanogenesis & 30 & $2.9928 \mathrm{E}-07$ & $2.1847 \mathrm{E}-05$ & 6.5239 \\
Focal adhesion (human) & 47 & $4.6332 \mathrm{E}-07$ & $2.5367 \mathrm{E}-05$ & 6.3341 \\
Protein processing in endoplasmic reticulum & 41 & $6.5163 \mathrm{E}-07$ & $2.8541 \mathrm{E}-05$ & 6.1860 \\
Long-term potentiation & 23 & $8.2838 \mathrm{E}-07$ & $3.0236 \mathrm{E}-05$ & 6.0818 \\
Calcium signaling & 42 & $1.2489 \mathrm{E}-06$ & $3.9074 \mathrm{E}-05$ & 5.9034 \\
Neurotrophin signaling & 33 & $1.7810 \mathrm{E}-06$ & $4.8755 \mathrm{E}-05$ & 5.7494 \\
Adherens junction & 23 & $3.1867 \mathrm{E}-06$ & $7.7544 \mathrm{E}-05$ & 5.4967 \\
Pathways in cancer & 64 & $3.5835 \mathrm{E}-06$ & $7.8478 \mathrm{E}-05$ & 5.4457 \\
\hline
\end{tabular}

* Number of genes associated with the corresponding pathway.

** Enrichment $p$-value of the corresponding pathway as determined by Fisher's exact test.

*** False discovery rate of the corresponding pathway.

**** Enrichment score value of the corresponding pathway, expressed as -log10( $p$-value). 


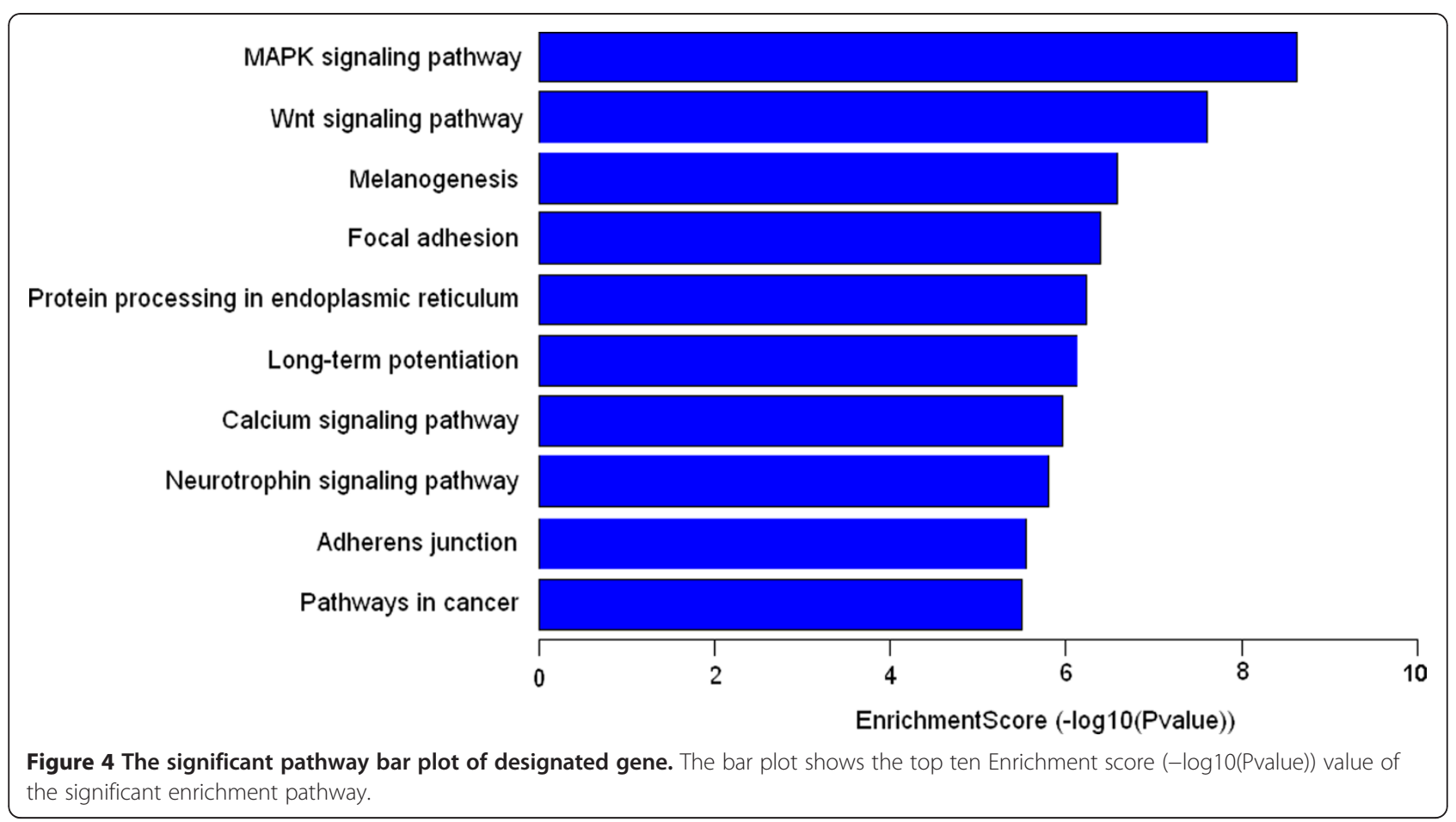

genome. Our findings may provide new insights towards understanding the mechanisms underlying the ability of $\mathrm{HBc}$ to regulate host gene transcription.

The ChIP technique has proven to be a powerful tool for detecting protein-DNA interactions in living cells, and it is currently the gold standard procedure for identifying target genes of a given transcription factor of interest. Over the last several years, great strides have been made in expanding the use of ChIP from a one gene-at-a-time approach to a global type of analysis by hybridizing samples to genomic microarrays (i.e., ChIP-on-chip assay) [26]. In such a study, Dere et al. previously identified 1896 of the 2,3,7,8-tetrachlorodibenzo$\mathrm{p}$-dioxin (TCDD)-responsive genes in mouse liver by using microarray analysis [27]. Most regions were enriched 5.7 -fold with the fold-enrichment values ranging from 1.7- to 111.4-fold [27]. Using ChIP-chip technique, Koudritsky and Domany showed that the number of target genes of each studied transcription factor (TF) ranged from a few hundred to several thousand [28]. Consistent

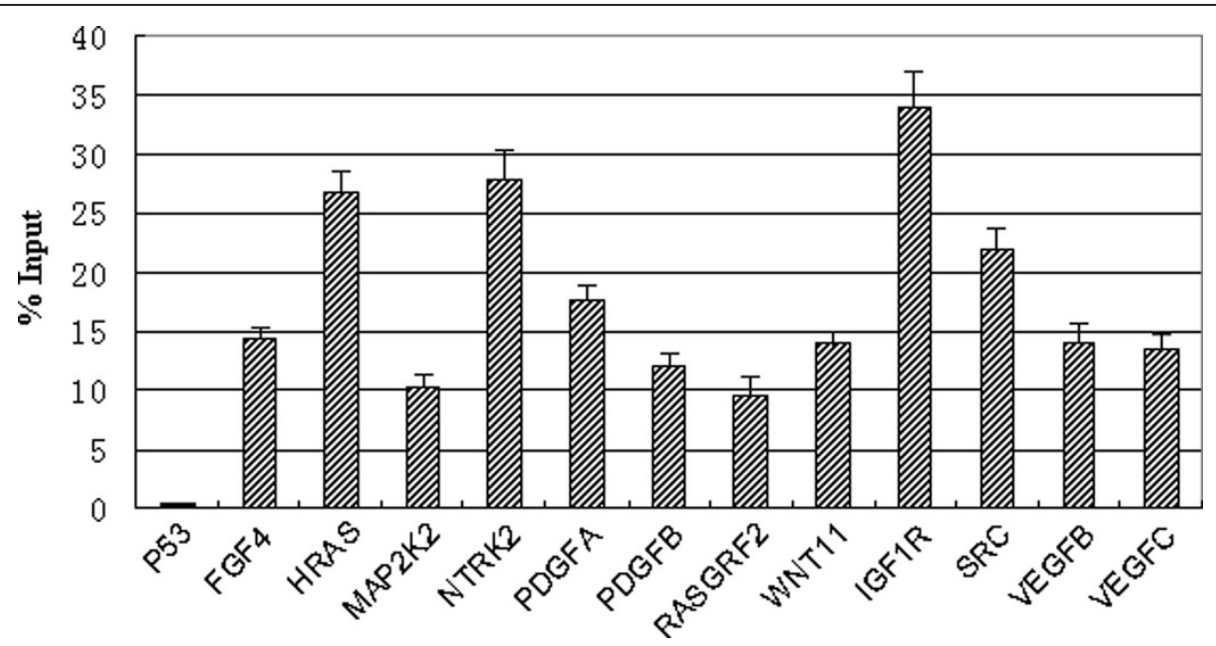

Figure 5 The results of ChIP-qPCR analysis of HBV core protein binding to host gene promoters. The promoter sequence percent of output DNA to input DNA (\% input) was detected using qPCR of ChIP samples. The p53 gene promoter was used as the negative control. Data are expressed as mean \pm SD of individual samples from three independent experiments. 


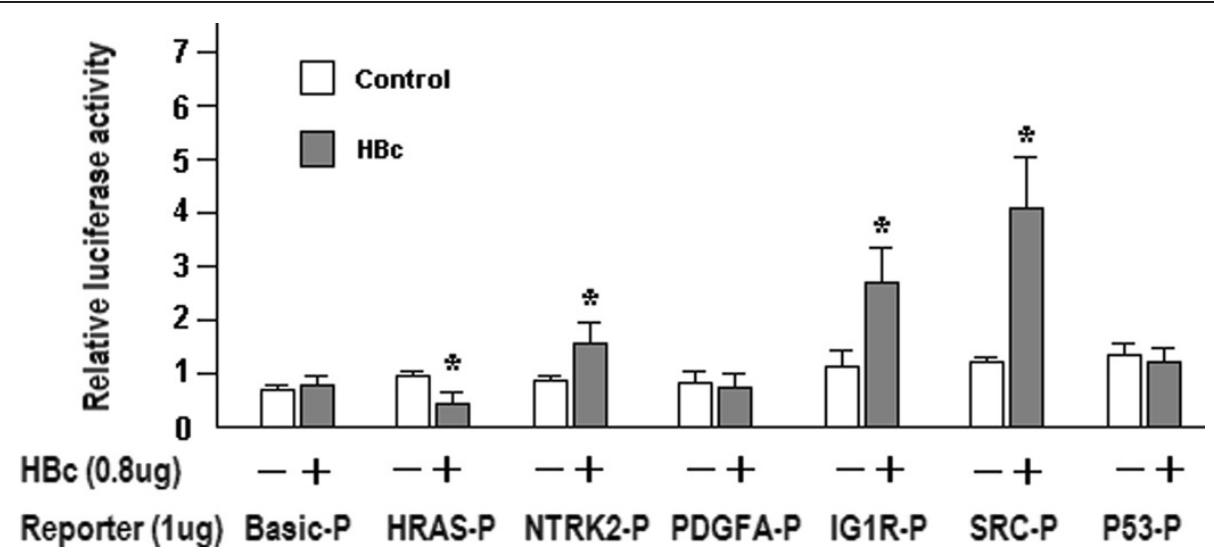

Figure 6 Effects of HBV core protein binding to host gene promoters. Relative luciferase activity was measured in cells co-transfected with one of six tumor pathogenesis-associated genes and the HBC gene. The pGL3-basic plasmid was used as the negative control. The vertical axis represents the relative proportion of luciferase activity of (cells co-transfected with $\mathrm{HBC}$ expression vector)/(cells transfected without $\mathrm{HBC}$ expression vector). Data are expressed as mean $\pm S D,{ }^{*} \mathrm{P}<0.05$. Basic-P, $\mathrm{pGL3}$-basic vectors; HRAS-P, NTRK2-P, PDGFA-P, IGF1R-P, SRC-P and P53-P represent the pGL3-basic-constructed plasmids for the promoters of HRAS, NTRK2, PDGFA, IGF1R, SRC and P53 genes, respectively.

with these data, $3100 \mathrm{HBc}$-immunoprecipitated host promoter regions were found to be enriched by at least 2-fold in HBc-infected tissues $(p<0.05$, compared with control). Moreover, 2883 of 3100 gene promoters were found in all of the four pooled samples investigated by ChIP-on-chip, which indicates that this approach has a good level of repeatability and the reliability. In our study, arrays representing 18028 promoter regions and CpG islands of the whole human genome were used in combination with HBV HBc-based ChIP to identify putative targets of this viral factor. We also identified 266 HBc-bound gene promoters that were different among the four pooled samples. Such apparently inconsistent findings may reflect background differences among the pooled ChIP samples. We found it necessary to use pooled samples for the ChIP-on-chip assays since none of our ChIP samples provided enough DNA for genomic microarray analysis. The practice of pooling ChIP samples for ChIP-on-chip is very common, but is known to produce very high background when the samples are analyzed on genomic tiling arrays [29]. To verify the reliability of our ChIP-on-chip results, the method of ChIP-qPCR was used to detect the promoter sequences of randomly selected genes in samples of our output DNA. Our results confirmed that $\mathrm{HBc}$ can bind to the promoters of all 12 genes evaluated, including the MxA gene, which has been demonstrated as a bona fide target of $\mathrm{HBc}$ and was used as a positive control in our study $[12,14]$.

The underlying mechanisms of the interaction between $\mathrm{HBc}$ and the human genome are poorly understood. $\mathrm{HBC}$ is a 183 residue protein with two domains: the N-terminal 144 amino acid domain that is sufficient for self-assembly into capsid particles, and the Cterminal arginine-rich domain, which shares a high similarity to protamine and functions as a nucleic acidbinding domain $[16,30]$. Although the C-terminal arginine-rich domain has been identified as imporatnt for $\mathrm{HBc}$ binding to pregenome RNA or genome DNA [31], no specific target sequence for the DNA-binding domain of $\mathrm{HBc}$ has been determined. Thus, while we know that the C-terminal arginine-rich domain can bind HBVspecific nucleotides in vivo, we have yet to determine the mechanisms mediating this binding specificity. However, based on the fact that HBV core particles specifically bind to the HBV pregenome or genome in vivo, we inferred that the binding specificity to the human genome might be mediated by the C-terminal arginine-rich domain, along with other regulatory regions in the $\mathrm{N}$ terminal assembly domain. Previous studies have shown that a signal for nuclear transport is located near the carboxy termini of $\mathrm{HBc}$, in the arginine-rich domain. This signal is comprised of a set of two direct PRRRRSQS repeats and is highly conserved among mammalian hepadnaviruses [32]. Recently, one study used a novel GST-fusion protein-based gel shift method to demonstrate that the C-terminal arginine-rich domain alone is capable of binding to DNA in a sequence-independent manner [33]. In addition, another study showed that HBc can enhance host NF-kB DNA-binding ability [34], which suggested that $\mathrm{HBc}$ may bind to and interact with other host nuclear proteins to enhance or inhibit their transcriptional activator functions. The large number of potential binding targets of $\mathrm{HBC}$ that were identified in our genome-wide analysis of human promoters supports the notion that $\mathrm{HBC}$ may utilize more than one of these proposed mechanisms. However, further study is needed to clarify this issue.

Previous studies have shown that HBV DNA integration and/or expression of HBV proteins may have direct 
effects on host cellular functions [35]. However, the roles of interaction between $\mathrm{HBV}$ proteins, especially $\mathrm{HBc}$, and the human genome have not been well studied. Locarnini et al. [36] examined the effect of the HBV core protein on cellular gene expression in the hepatoma Huh-7 cell line by using a commercial high-density oligonucleotide array (Affymetrix Hu95A GeneChip). They found that only five genes had differential mRNA expression that was greater than three-fold at day 7 post-HBc expression. Among these five genes, four were down-regulated (by $3 \sim 15$-fold) and included genes whose encoded products affect intermediary metabolism, cell surface receptors, and intracellular signaling. The fifth, a cytokine gene, was up-regulated. In contrast, our study utilized a microarray containing the genome-wide set of human gene promoters to search for potential binding sites of $\mathrm{HBc}$, and identified over 3000 putative functional targets. Even though the full panel of these promoters has yet to be confirmed, the diverse spectrum of genes represented suggests that $\mathrm{HBc}$ may have multiple functions in regulating HBV pathogenesis and survival in the human host.

It is likely that $\mathrm{HBc}$ binding does not exert significant regulatory effects on every gene for which its promoter is bound. For some or many genes, HBc may merely mediate a slight modulation of the transcriptional activity. However, the cumulative effect of slight transcriptional modifications across the genome may impact overall cellular function. In our study, we found that $\mathrm{HBc}$ can bind to 64 gene promoters of the MAPK pathways, and 41 gene promoters of the $\mathrm{Wnt} / \beta$-catenin signaling pathways. These two pathways are known to be critically involved in the development of HBV-related hepatocellular carcinoma. The MAPK pathways regulate a variety of normal human cellular activities, including proliferation, differentiation, survival, and death. As such, the components of MAPK signaling play a key role in several steps of tumorigenesis, including cancer cell proliferation, migration, and invasion [37]. Meanwhile, the Wnt/ $\beta$-catenin signaling pathway has emerged as a critical player in both the development of normal liver and as an oncogenic driver in hepatocellular carcinoma [38]. As described above, the accumulation of slight effects from $\mathrm{HBc}$ binding to many gene promoters may produce sizeable effects on host cellular functions, possibly increasing a cell's susceptibility to harmful factors, such as carcinogens [39]. Certainly, further studies will be needed to verify this theory and define the detailed factor(s) and step(s) that HBc works through, or that work through $\mathrm{HBc}$, to regulate host gene transcription.

We investigated the functional relevance of HBV core protein binding to five of the genes whose promoters were identified in the ChIP-on-chip analysis. Transcriptional activity assays indicated that SRC, IGF1R, and
NTRK2 genes were up-regulated by more than 2-fold when co-transfected with HBc. The HRAS gene, however, was down-regulated in the presence of HBc. SRC is the best characterized member of the family of nine tyrosine kinases that regulates cellular responses to extracellular stimuli, and activated mutants of SRC are oncogenic [40]. Among these four genes, SRC showed the greatest change in gene expression in $\mathrm{HBc}$ co-transfections, which may be related to the SRC gene promoter sequence that contains a potential NF- $\mathrm{kB}$ binding sequence (5/-GGGAAAATCC-3/). Thus, HBc might enhance the NF- $\mathrm{kB}$ DNA-binding ability on SRC [34]. However, it should be noted that the possible roles of the HBV core protein to the transcriptional activity of only small number of gene promoters were evaluated in our study; more sensitive and accurate high-throughput DNA-protein interaction and gene expression analysis techniques should be used in further studies.

\section{Conclusion}

In conclusion, regardless of the mechanisms, the data of our study clearly demonstrate that $\mathrm{HBc}$ not only has the ability to bind to a large number of gene promoters in the human genome, but also has the ability to disrupt cellular gene expression of HBV-infected hepatocytes. Although further studies are needed to investigate the possible role of $\mathrm{HBc}$ in regulating the transcriptional activity of all cellular genes that $\mathrm{HBc}$ can bind, our study provides key insights into the detailed factor(s) and step (s) that HBc works through, or that work through HBc, to regulate host gene transcription. This information provides a new perspective towards understanding the mechanisms of HBV-related liver diseases, such as liver chronicity, fibrogenesis, and carcinogenesis. Manipulation of the transcriptional profile in HBV-infected hepatocytes may represent a key pathogenic mechanism of HBV infection.

\section{Additional files}

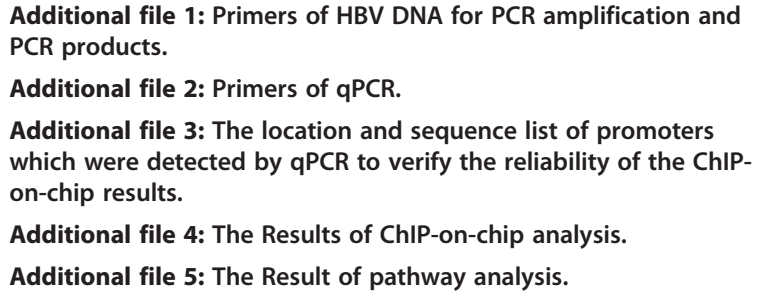

Additional file 3: The location and sequence list of promoters which were detected by qPCR to verify the reliability of the ChIPon-chip results.

Additional file 4: The Results of ChIP-on-chip analysis.

Additional file 5: The Result of pathway analysis.

\section{Competing interests}

The authors declare that they have no competing interests.

\section{Authors' contributions}

YG designed this study, performed the ChIP experiment, reporter gene analysis, and statistical analysis, and prepared this manuscript. WK and YL collected clinical samples and patient information. WK examined the HBV 
DNA level and HBV indexes from serum samples and also revised the manuscript. $X L$ and $A X$ performed the $\mathrm{GPCR}$ experiment and analyzed the data. YL, JZ, and JZ participated in the reporter gene analysis. ZY helped with manuscript revision. All authors read and approved the final manuscript.

\section{Financial disclosure}

This study was supported by grants from the 11th Five-Year Plan of China: the Project for Prevention and Treatment of AIDS, Viral Hepatitis and Other Major Infectious Diseases (No. 2009ZX10004-311-004), the Natural Science Basic Research Program of Shaanxi Province of China (No. 2009JM4005) and the National Natural Science Foundation of China (No. 31271394).

\section{Acknowledgements}

The authors are grateful to Weihua Wang Dr, Xiaochang Xue Dr, An Xiang M. S., Biyun Li (technician), and Xiyan Zhang (technician) (Institute of Genetic Diagnosis, Fourth Military Medical University, Xi'an, China) for excellent technical assistance. In addition, the authors would like to thank the editors at Medjaden Bioscience Limited for their critical reading and helpful comments on the manuscript.

\section{Author details}

${ }^{1}$ State Key Laboratory of Cancer Biology, Department of Pharmacogenomics, School of Pharmacy, Fourth Military Medical University, 169 West Changle Road, Xi'an 710032, China. ${ }^{2}$ Department of Clinical Laboratory, Affiliated Hospital of Xi'an Medical University, Xi'an 710077, China. ${ }^{3}$ Department of Infectious Diseases, 323 Hospital, Xi'an 710000, China.

Received: 12 March 2012 Accepted: 15 October 2012

Published: 22 October 2012

\section{References}

1. Ganem D, Prince AM: Hepatitis B virus infection-natural history and clinical consequences. N Engl J Med 2004, 350:1118-1129.

2. Shepard CW, Simard EP, Finelli L, Fiore AE, Bell BP: Hepatitis B virus infection: epidemiology and vaccination. Epidemiologic reviews 2006, 28:112-125.

3. Bock CT, Schwinn S, Locarnini S, Fyfe J, Manns MP, Trautwein C, Zentgraf H: Structural organization of the hepatitis B virus minichromosome. J Mol Biol 2001, 307:183-196.

4. Haybaeck J, Zeller N, Wolf MJ, Weber A, Wagner U, Kurrer MO, Bremer J, lezzi G, Graf R, Clavien PA, Thimme R, Blum H, Nedospasov SA, Zatloukal K, Ramzan M, Ciesek S, Pietschmann T, Marche PN, Karin M, Kopf M, Browning $J$, Aguzzi A, Heikenwalder M: A lymphotoxin-driven pathway to hepatocellular carcinoma. Cancer Cell 2009, 16:295-308.

5. Brechot C: Pathogenesis of hepatitis $B$ virus-related hepatocellular carcinoma: old and new paradigms. Gastroenterology 2004, 127:S56-S61.

6. Lee AT, Lee CG: Oncogenesis and transforming viruses: the hepatitis B virus and hepatocellularcarcinoma-the etiopathogenic link. Front Biosci 2007, 12:234-245.

7. Murakami S: Hepatitis B virus X protein: structure, function and biology Intervirology 1999, 42:81-99.

8. Petit MA, Pillot J: $\mathrm{HBC}$ and $\mathrm{HBe}$ antigenicity and DNA-binding activity of major core protein P22 in hepatitis B virus core particles isolated from the cytoplasm of human liver cells. J Virol 1985, 53:543-551.

9. Michalak T, Nowoslawski A: Crystalline aggregates of hepatitis B core particles in cytoplasm of hepatocytes. Intervirology 1982, 17:247-252.

10. Du J, Liang X, Liu Y, Qu Z, Gao L, Han L, Liu S, Cui M, Shi Y, Zhang Z, et al: Hepatitis $B$ virus core protein inhibits TRAIL-induced apoptosis of hepatocytes by blocking DR5 expression. Cell death and differentiation 2009, 16:219-229.

11. Kim JH, Kang S, Kim J, Ahn BY: Hepatitis B virus core protein stimulates the proteasome-mediated degradation of viral X protein. J Virol 2003, 77:7166-7173.

12. Kwon JA, Rho HM: Transcriptional repression of the human p53 gene by hepatitis B viral core protein (HBc) in human liver cells. Biol Chem 2003, 384:203-212.

13. Elmore LW, Hancock AR, Chang SF, Wang XW, Chang S, Callahan CP, Geller DA, Will $H$, Harris CC: Hepatitis B virus $X$ protein and $p 53$ tumor suppressor interactions in the modulation of apoptosis. Proc Natl Acad Sci US A 1997, 94:14707-14712.
14. Fernandez M, Quiroga JA, Carreno V: Hepatitis B virus downregulates the human interferon-inducible MxA promoter through direct interaction of precore/core proteins. J Gen Virol 2003, 84:2073-2082.

15. Liao $\mathrm{W}, \mathrm{Ou} \mathrm{JH}$ : Phosphorylation and nuclear localization of the hepatitis $B$ virus core protein: significance of serine in the three repeated SPRRR motifs. J Virol 1995, 69:1025-1029.

16. Zheng J, Schodel F, Peterson DL: The structure of hepadnaviral core antigens. Identification of free thiols and determination of the disulfide bonding pattern. J Biol Chem 1992, 267:9422-9429.

17. Gallina A, Bonelli F, Zentilin L, Rindi G, Muttini M, Milanesi G: A recombinant hepatitis $B$ core antigen polypeptide with the protamine-like domain deleted self-assembles into capsid particles but fails to bind nucleic acids. J Virol 1989, 63:4645-4652.

18. Ren B, Robert F, Wyrick JJ, Aparicio O, Jennings EG, Simon I, Zeitlinger J, Schreiber J, Hannett N, Kanin E, et al: Genome-wide location and function of DNA binding proteins. Science 2000, 290:2306-2309.

19. Chinese Society of Hepatology CMACSoID, Chinese Medical Association: Guideline on prevention and treatment of chronic hepatitis B in China (2005). Chin Med J (Engl) 2007, 120:2159-2173.

20. Yan Z, Tan W, Zhao W, Dan Y, Wang X, Mao Q, Wang Y, Deng G: Regulatory polymorphisms in the IL-10 gene promoter and HBV-related acute liver failure in the Chinese population. J Viral Hepat 2009, 16:775-783.

21. Hatzis $P$, van der Flier $L G$, van Driel MA, Guryev V, Nielsen F, Denissov S, Nijman IJ, Koster J, Santo EE, Welboren W, et al: Genome-wide pattern of TCF7L2/TCF4 chromatin occupancy in colorectal cancer cells. Mol Cell Biol 2008, 28:2732-2744

22. Pathak S, D'Souza R, Ankolkar M, Gaonkar R, Balasinor NH: Potential role of estrogen in regulation of the insulin-like growth factor2-H19 locus in the rat testis. Mol Cell Endocrinol 2010, 314:110-117.

23. Kim SK, Jang SK, Rho HM: Effect of frameshift mutation in the pre-C region of hepatitis B virus on the X and C genes. J Gen Virol 1994, 75(Pt 4):917-923.

24. Kanehisa M, Goto S, Kawashima S, Okuno Y, Hattori M: The KEGG resource for deciphering the genome. Nucleic Acids Res 2004, 32:D277-D280.

25. Guo YH, Li YN, Zhao JR, Zhang J, Yan Z: HBc binds to the CpG islands of HBV cccDNA and promotes an epigenetic permissive state. Epigenetics: official journal of the DNA Methylation Society 2011, 6:720-726.

26. Buck MJ, Lieb JD: ChIP-chip: considerations for the design, analysis, and application of genome-wide chromatin immunoprecipitation experiments. Genomics 2004, 83:349-360.

27. Dere E, Lo R, Celius T, Matthews J, Zacharewski TR: Integration of genomewide computation DRE search, AhR ChIP-chip and gene expression analyses of TCDD-elicited responses in the mouse liver. BMC Genomics 2011, 12:365.

28. Koudritsky M, Domany E: Positional distribution of human transcription factor binding sites. Nucleic Acids Res 2008, 36:6795-6805.

29. O'Geen H, Nicolet CM, Blahnik K, Green R, Farnham PJ: Comparison of sample preparation methods for ChIP-chip assays. Biotechniques 2006, 41:577-580

30. Blanchet M, Sureau C: Analysis of the cytosolic domains of the hepatitis B virus envelope proteins for their function in viral particle assembly and infectivity. J Virol 2006, 80:11935-11945.

31. Kock J, Nassal M, Deres K, Blum HE, von Weizsacker F: Hepatitis B virus nucleocapsids formed by carboxy-terminally mutated core proteins contain spliced viral genomes but lack full-size DNA. J Virol 2004, 78:13812-13818.

32. Yeh $C T$, Liaw YF, Ou JH: The arginine-rich domain of hepatitis B virus precore and core proteins contains a signal for nuclear transport. J Virol 1990, 64:6141-6147.

33. Lu L, Liu W, Yang $X$ : A novel method for characterizing the multifunctional C-terminal domain of the Hepadnavirus core protein. J Virol Methods 2009, 158:195-198.

34. Kwon JA, Rho HM: Hepatitis B viral core protein activates the hepatitis B viral enhancer II/pregenomic promoter through the nuclear factor kappaB binding site. Biochemistry and cell biology = Biochimie et biologie cellulaire 2002, 80:445-455.

35. Chemin I, Zoulim F: Hepatitis B virus induced hepatocellular carcinoma. Cancer Lett 2009, 286:52-59.

36. Locarnini S, Shaw T, Dean J, Colledge D, Thompson A, Li K, Lemon SM, Lau $\mathrm{GG}$, Beard MR: Cellular response to conditional expression of the 
hepatitis B virus precore and core proteins in cultured hepatoma (Huh-7) cells. J Clin Virol 2005, 32:113-121.

37. Kim EK, Choi EJ: Pathological roles of MAPK signaling pathways in human diseases. Biochim Biophys Acta 2010, 1802:396-405.

38. Fatima S, Lee NP, Luk JM: Dickkopfs and Wnt/beta-catenin signalling in liver cancer. World journal of clinical oncology 2011, 2:311-325.

39. Zheng Y, Chen WL, Louie SG, Yen TS, Ou JH: Hepatitis B virus promotes hepatocarcinogenesis in transgenic mice. Hepatology 2007, 45:16-21.

40. Brown MT, Cooper JA: Regulation, substrates and functions of src. Biochim Biophys Acta 1996, 1287:121-149.

doi:10.1186/1471-2164-13-563

Cite this article as: Guo et al.: Hepatitis B viral core protein disrupts human host gene expression by binding to promoter regions. $B M C$ Genomics 2012 13:563.

\section{Submit your next manuscript to BioMed Central and take full advantage of:}

- Convenient online submission

- Thorough peer review

- No space constraints or color figure charges

- Immediate publication on acceptance

- Inclusion in PubMed, CAS, Scopus and Google Scholar

- Research which is freely available for redistribution 